\title{
The curious activity in the nucleus of NGC 4151: jet interaction causing variability?
}

\author{
D.R.A. Williams, ${ }^{1,2 \star ~ R . D . B a l d i, ~}{ }^{2,4,5}$ I.M. McHardy ${ }^{2}$ R.J. Beswick, ${ }^{3}$ F. Panessa, ${ }^{5}$ \\ D. May, ${ }^{6}$ J. Moldón, ${ }^{3,7}$ M.K. Argo, ${ }^{3,8}$ G. Bruni, ${ }^{5}$, B.T. Dullo, ${ }^{9}$ J.H. Knapen, ${ }^{10,11,12}$ \\ E. Brinks, ${ }^{13}$ D.M. Fenech, ${ }^{14}$ C.G. Mundell,,${ }^{15}$ T.W.B. Muxlow,${ }^{3}$ M. Pahari, ${ }^{2}$ \\ J. Westcott ${ }^{13}$ \\ ${ }^{1}$ Department of Physics, University of Oxford, Denys Wilkinson Building, Keble Road, Oxford, OX1 3RH, UK \\ ${ }^{2}$ School of Physics and Astronomy, University of Southampton, Southampton, SO17 1BJ, UK \\ ${ }^{3}$ Jodrell Bank Centre for Astrophysics, School of Physics and Astronomy, The University of Manchester, Manchester, M13 9PL, UK \\ ${ }^{4}$ Dipartimento di Fisica, Universitá degli Studi di Torino, via Pietro Giuria 1, 10125 Torino, Italy \\ ${ }^{5}$ INAF - Istituto di Astrofisica e Planetologia Spaziali, via Fosso del Cavaliere 100, I-00133 Roma, Italy \\ ${ }^{6}$ Instituto de Astronomia, Geofísica e Ciências Atmosféricas, Universidade de São Paulo, 05508-090 São Paulo, SP, Brazil \\ ${ }^{7}$ Instituto de Astrofísica de Andalucía (IAA, CSIC), Glorieta de las Astronomía, s/n, E-18008 Granada, Spain \\ ${ }^{8}$ University of Central Lancashire, Jeremiah Horrocks Institute Preston, UK PR1 2HE \\ ${ }^{9}$ Departamento de Física de la Tierra y Astrofísica, Instituto de Física de Partículas y del Cosmos IPARCOS, \\ Universidad Complutense de Madrid, E-28040 Madrid, Spain \\ ${ }^{10}$ Instituto de Astrofísica de Canarias, Vía Láctea S/N, E-38205 La Laguna, Spain \\ ${ }^{11}$ Departamento de Astrofísica, Universidad de La Laguna, E-38206 La Laguna, Spain \\ ${ }_{12}$ Astrophysics Research Institute, Liverpool John Moores University, IC2, Liverpool Science Park, 146 Brownlow Hill, \\ Liverpool, L3 $5 R F$, UK \\ ${ }^{13}$ Centre for Astrophysics Research, University of Hertfordshire, College Lane, Hatfield, AL10 9AB, UK \\ ${ }^{14}$ Astrophysics Group, Cavendish Laboratory, 19 J. J. Thomson Avenue, Cambridge CB3 OHE, UK \\ ${ }^{15}$ Department of Physics, University of Bath, Claverton Down, Bath, BA2 7AY, UK
}

Accepted XXX. Received YYY; in original form ZZZ

\begin{abstract}
A key characteristic of many active galactic nuclei (AGN) is their variability, but its origin is poorly understood, especially in the radio domain. Williams et al. (2017) reported a $\sim 50$ per cent increase in peak flux density of the AGN in the Seyfert galaxy NGC 4151 at $1.5 \mathrm{GHz}$ with the e-MERLIN array. We present new high resolution eMERLIN observations at $5 \mathrm{GHz}$ and compare these to archival MERLIN observations to investigate the reported variability. Our new observations allow us to probe the nuclear region at a factor three times higher-resolution than the previous e-MERLIN study. We separate the core component, $\mathrm{C} 4$, into three separate components: $\mathrm{C} 4 \mathrm{~W}$, $\mathrm{C} 4 \mathrm{E}$ and $\mathrm{X}$. The AGN is thought to reside in component $\mathrm{C} 4 \mathrm{~W}$, but this component has remained constant between epochs within uncertainties. However, we find that the Eastern-most component, C4E, has increased in peak flux density from $19.35 \pm 1.10$ to $37.09 \pm 1.86 \mathrm{mJy} /$ beam, representing a $8.2 \sigma$ increase on the MERLIN observations. We attribute this peak flux density increase to continued interaction between the jet and the emission line region (ELR), observed for the first time in a low-luminosity AGN such as NGC 4151. We identify discrete resolved components at $5 \mathrm{GHz}$ along the jet axis, which we interpret as areas of jet-ELR interaction.
\end{abstract}

Key words: galaxies: active - galaxies: individual: NGC 4151 - galaxies: jets - galaxies: nuclei - quasars: emission lines - galaxies: Seyfert.

\section{INTRODUCTION}

^ E-mail: david.williams@physics.ox.ac.uk

Astrophysical radio jets are a common signature of the accretion process onto compact objects such as super-massive

(C) 2015 The Authors 
black holes (SMBHs). Jets are responsible for mechanical "feedback" into their surroundings, triggering and quenching star formation as well as regulating galaxy evolution (e.g. Fabian 2012; Morganti et al. 2013). An accreting SMBH is known as an active galactic nucleus (AGN). The most powerful AGN such as those in Fanaroff-Riley Type I and Type II radio galaxies (Fanaroff \& Riley 1974) host jets that can be launched at close to the speed of light (Begelman et al. 1984; Urry \& Padovani 1995). However, in low-luminosity AGN (LLAGN), and radio-quiet AGN (defined by Kellermann et al. 1989, as $\mathrm{L}_{\text {radio }} / \mathrm{L}_{\text {opt }}<10$ ), compact radio emission and jet-like features have been observed, though often less collimated and less powerful (e.g. Nagar et al. 2000; Baldi et al. 2018). In such sources, the presence of compact nuclear radio emission has been attributed to a variety of different processes (see Panessa et al. 2019, for a review), including free-free emission/absorption (Ulvestad \& Ho 2001; Gallimore et al. 2004), a combination of a compact radio jet plus an advection dominated accretion flow (ADAF, Falcke \& Markoff 2000), a standard geometrically-thin opticallythick accretion disk (Shakura \& Sunyaev 1973; Ghisellini et al. 2004) or outflowing material at relatively low velocities (Giroletti et al. 2017; Laor et al. 2019). To resolve these different emission processes in nearby LLAGN, high-resolution radio interferometers such as e-MERLIN are necessary to separate the core component from circum-nuclear star formation.

Radio variability has been observed in luminous AGN (e.g. Hufnagel \& Bregman 1992) and is a useful tool for discriminating between the different radio emission mechanisms (Panessa et al. 2019). In some cases, the radio variability is found to be related to activity in the jet, rather than in the self-absorbed synchrotron emitting core component attributed to the AGN (e.g. in NGC 1052, Baczko et al. 2016, 2019). However, due to their intrinsic radio-weakness, variability studies of LLAGN have been limited to small samples of the best known sources, predominantly Seyfert galaxies (Wrobel 2000; Nagar et al. 2002; Mundell et al. 2009; Bell et al. 2011). Variability of up to 40 per cent has been reported in half of a sample of Seyfert galaxies over a seven year period (Mundell et al. 2009), while almost no variability has been reported in some other cases (e.g. NGC 4051, Jones et al. 2011). Shorter, month-long variability has been detected in the Seyfert galaxy NGC 5548, (Wrobel 2000), and weak radio variability has also been detected on a few days timescales in radio-quiet AGN systems (Anderson \& Ulvestad 2005; Baldi et al. 2015). However, only a handful variability studies have been undertaken at decade-long timescales or longer and in most cases the variability has been attributed to the emission from the core.

Recently, a change of radio flux density has been reported for the core of the radio-quiet Seyfert galaxy NGC 4151. In MERLIN and e-MERLIN resolution maps (Pedlar et al. 1993; Williams et al. 2017) the components are labelled from $\mathrm{C} 1$ to $\mathrm{C} 5$ and the unresolved core is $\mathrm{C} 4$. Higher resolution VLBI observations (Ulvestad et al. 1998; Mundell et al. 2003; Ulvestad et al. 2005a) resolve some of these components into multiple smaller components. The VLBI components are labelled A to I. The correspondence between the VLBI and MERLIN/e-MERLIN components is shown in Fig.1 of Mundell et al. (2003). In this paper we use both conventions, which we also illustrate in Fig.1 here.
The central component, C4, unresolved in e-MERLIN observations at 150 mas at $1.5 \mathrm{GHz}$, increased in peak flux density from $\sim 37 \mathrm{mJy} /$ beam (Mundell et al. 1995 ) to $\sim 67 \mathrm{mJy} /$ beam (Williams et al. 2017), over a period of 22 years. We obtained new $5 \mathrm{GHz}$ observations of NGC 4151 with e-MERLIN (proposal ID: CY6219), which probes angular resolutions up to 50 mas, improving the angular resolution of the observations of Williams et al. (2017) by a factor of three. This allows us to investigate the varying radio components in the galaxy core at higher imaging fidelity.

We assume a distance to NGC 4151 of $19 \mathrm{Mpc}$, (Hönig et al. 2014). This paper is structured as follows: In Section 2 we describe the new e-MERLIN data and reduction procedures. In Section 3 we show our results and in Section 4, discuss their implications on the AGN core position in NGC 4151 and Section 5 lists our conclusions.

\section{OBSERVATIONS AND DATA REDUCTION}

NGC 4151 was observed on 2017 July 19 and correlated at Jodrell Bank, using all e-MERLIN stations except the Lovell telescope. The observing set-up was centred at $5.07 \mathrm{GHz}$, using a total bandwidth of $512 \mathrm{MHz}$ and split into four spectral windows (spws). The target field was observed for 3.15 hours in 6.5 minute scans, interleaved with 2.5 minute scans of the phase calibrator NVSSJ115354+403652 (J1153+4036, RA: $11^{\mathrm{h}} 53^{\mathrm{m}} 54.6594^{\mathrm{s}}$, Dec: $\left.+40^{\circ} 36^{\prime} 52.617^{\prime \prime}\right)$. The standard e-MERLIN band pass (OQ208) and flux density (3C286) calibrators were observed at the beginning of the observing run for 14 minutes and 25 minutes respectively. Unfortunately, the data for the Mark II telescope did not provide any phase information and thus the antenna was flagged; Pickmere was used as a reference antenna. The remaining five antennas generally showed good phase stability. The data were calibrated with version 0.7 .9 of the CASA e-MERLIN pipeline ${ }^{1}$. The CASA e-MERLIN pipeline is similar to the previous AIPS data reduction procedure outlined in Williams et al. (2017), (see also Westcott et al. 2017; Baldi et al. 2018; Williams et al. 2019) for e-MERLIN continuum data: the pipeline performs standard calibration of e-MERLIN data by flagging radio frequency interference (RFI) using AOFLAGGER (Offringa et al. 2012), solving the delays of the antennas, performing phase and amplitude calibration, bootstrapping the flux density scaling, fitting and applying a band pass and making preliminary images and diagnostic plots, ready for further imaging and self calibration of the target fields.

The imaging procedures were conducted in CASA using the CASA task TCLEAN. We performed a standard phase self-calibration on the target source and found some amplitude errors on the shortest baselines between the Pickmere and Darnhall telescopes, which we then flagged from the data. By removing this information, we are less sensitive to the diffuse emission along the jet, but are still able to resolve small compact components of emission. When no further improvements through self-calibration could be made, a final image was produced with natural weighting. An rms noise level of $70 \mu \mathrm{Jy} /$ beam was achieved for the e-MERLIN

1 Online documentation can be found at: https://github.com/e-merlin/CASA_e-MERLIN_pipeline/wiki 


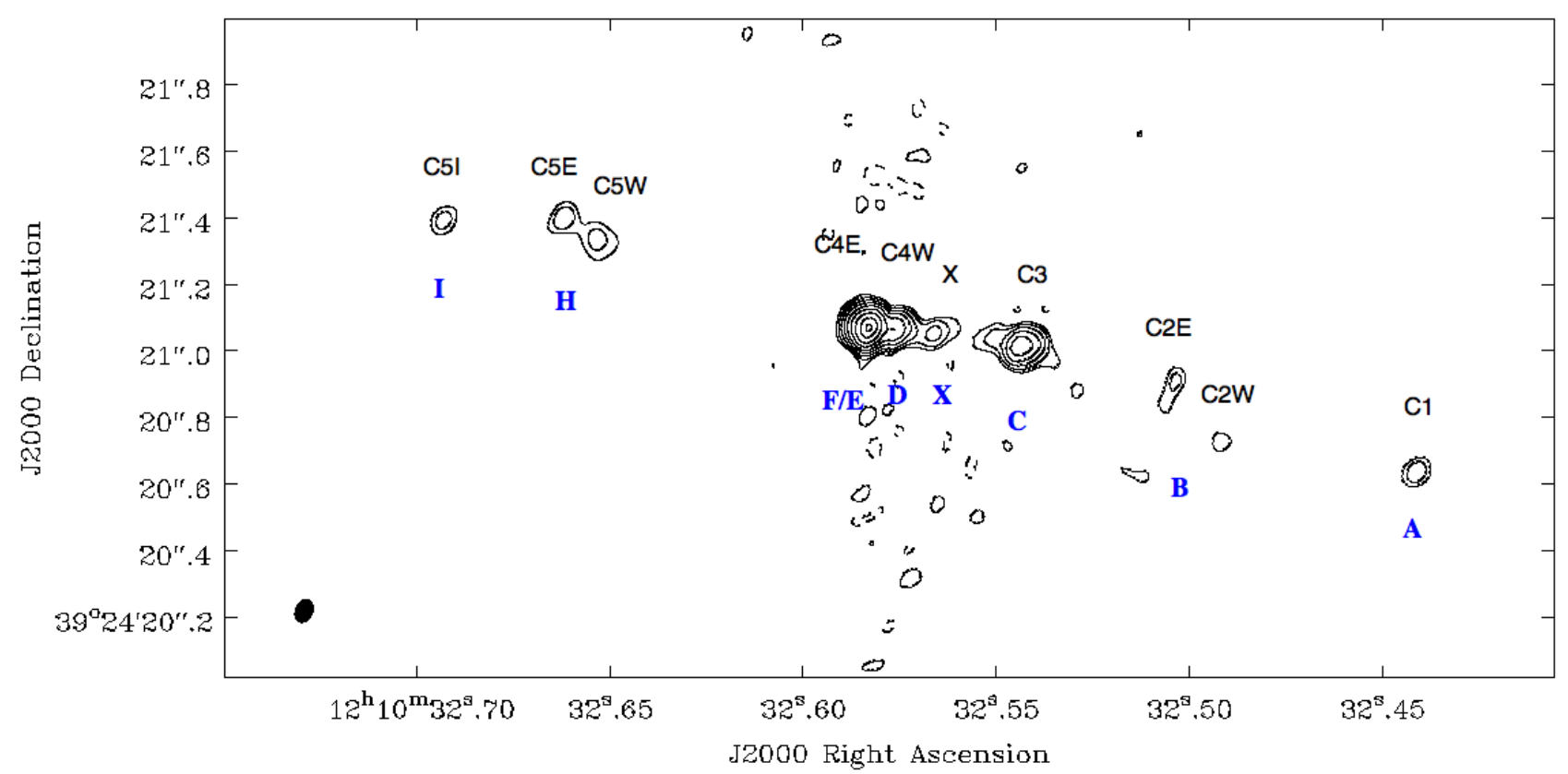

Figure 1. Naturally weighted full resolution e-MERLIN $5 \mathrm{GHz}$ image of the central $2.0^{\prime \prime} \times 3.75^{\prime \prime}(\sim 180 \times 340 \mathrm{pc})$ of NGC 4151 . The eMERLIN synthesized beam is shown as the filled ellipse in the bottom left hand corner: $0.07^{\prime \prime} \times 0.05^{\prime \prime}$ with a P.A. of $-24^{\circ}$. Contours are set at the rms noise level, $70 \mu \mathrm{Jy} /$ beam $\times-2.5,2.5,5,10,20,40,80,160,320,500$. The bold, blue, labels below (i.e., south of) the components (A to I) refer to the labels from higher resolution VLBA observations (Mundell et al. 2003). The black labels above (i.e., north of) the components ( $\mathrm{C} 1$ to $\mathrm{C} 5)$ refer to components from lower resolution MERLIN and e-MERLIN observations (Pedlar et al. 1993; Williams et al. 2017). The component properties are listed in Table 1.

data and was estimated from a region near the phase centre but not including the radio components of the jet. The rms noise regions were chosen so as not to encompass the areas directly north and south of the core region, which show some low-level artefacts in the image due to lack of $u v$-coverage on the shortest baselines. The full resolution image, with a synthesized beam of $0.05^{\prime \prime} \times 0.07^{\prime \prime}$ and a position angle (P.A.) of $-24^{\circ}$, is shown in Fig. 1. The P.A. of the full resolution image resulted in the major axis of the beam being perpendicular to the jet axis, allowing for identification of jet structures down to $0.05^{\prime \prime}$, corresponding to a linear scale of $\sim 4.6 \mathrm{pc}$.

\subsection{Archival MERLIN data}

NGC 4151 was observed by MERLIN, the predecessor to eMERLIN, on 1991 September 12 at $4.993 \mathrm{GHz}$ with a bandwidth of $7 \mathrm{MHz}$. These data are published in Pedlar et al. (1993) at a reduced resolution of 75 mas. We refer the reader to Pedlar et al. (1993) for the details of the 1991 observation and data reduction procedures. We obtained the calibrated Pedlar et al. archival data from Jodrell Bank with the goal of imaging them at the same resolution and $u v$-range as our new e-MERLIN data, to ensure that any changes of observed flux density found with the new data were significant (see next Section). The calibrated $u v$-dataset was examined and self calibrated in AIPS and exported into CASA file format for commensurate cleaning procedures with the e-MERLIN data.

\section{$2.2 u v$-matching and imaging the datasets}

To ensure that any changes in flux density are genuine and comparison between the two epochs is robust, we ensured that the e-MERLIN and MERLIN datasets used the same $u v$-information (see Section 2.1.3 of Williams et al. 2017, for details of the procedure performed for the $1.5 \mathrm{GHz}$ data). As the $u v$-coverage of the new e-MERLIN array is better than that of MERLIN, we first matched the $u v$-coverage of the two datasets, using the same $u v$-range $(400-3500 \mathrm{k} \lambda)$. This range was chosen to use all of the long baseline information to ensure the highest possible resolution images, but to remove amplitude errors found on the shortest baseline between Pickmere and Darnhall in the e-MERLIN data. As a consequence, this $u v$-range corresponds to angular scales of $0.05-0.5^{\prime \prime}$, which means the new e-MERLIN data are not sensitive to diffuse emission larger that $0.5^{\prime \prime}$ in size. We removed antennas from both datasets that only participated in one observation ${ }^{2}$. Finally, we fixed the restoring beam of both datasets to the same major and minor axes, as well as position angle. As the full resolution MERLIN data resulted in a circular beam of $0.05^{\prime \prime}$, we cleaned both datasets with a restoring beam of $0.07^{\prime \prime} \times 0.05^{\prime \prime}$ and a P.A. equal to that of

\footnotetext{
2 The now defunct Mark III (Wardle) antenna did not take part in the 1991 observations, and we removed the Mark II antenna from the 1991 data as it was not present in the 2017 e-MERLIN data.
} 
the full resolution e-MERLIN data, in order to preserve as much resolution along the jet axis as possible, while avoiding a super-resolution of either dataset.

The final MERLIN image reached an rms-noise value of $500 \mu \mathrm{Jy} /$ beam. This noise is higher than that reported in Pedlar et al. (1993) ( 100 $\mu \mathrm{Jy} /$ beam $)$ and is likely due to the reduced $u v$-range used in these data and removal of the Mark II telescope. As such, we are unable to make an image of the entire jet structure as the $u v$-range cut and lack of short spacing antennas means we are no longer sensitive to the diffuse emission along the jet axis shown by Pedlar et al. (1993). We show the MERLIN image of the detected components $\mathrm{C} 3$ and $\mathrm{C} 4$ in Fig. 2.We show the $u v$-limited e-MERLIN image for the same region of the jet in Fig. 3.

\subsection{Fitting components in the data}

The flux densities of all the components were extracted using the IMFIT task in CASA, which fits a two dimensional Gaussian to the regions of interest. For sources where the component profiles are merged or overlap, e.g. components $\mathrm{C} 4 \mathrm{E} / \mathrm{C} 4 \mathrm{~W}$, we fix the source size to that of the beam and fit both the positions and flux densities of the sources simultaneously. The flux density uncertainties obtained from the fitting procedure are added in quadrature to a 5 per cent flux density bootstrapping uncertainty for MERLIN/eMERLIN data (Rampadarath et al. 2018). We report the extracted flux densities and associated uncertainties from the $u v$-matched datasets and are reported in Table 1.

\subsection{Radio Spectra}

As an additional analysis, we split the e-MERLIN band into two halves, in order to create an in-band spectral index image using the CASA task IMMATH (grey-scale plot in Fig. 3). The spectral index is defined as $\mathrm{S}_{v} \propto v^{\alpha}$. As the e-MERLIN band at $5 \mathrm{GHz}$ is $512 \mathrm{MHz}$, there is a decrease in sensitivity across the band of $\sim 7$ per cent, assuming a spectral index of -0.7 is used, applicable for radio galaxies. To ensure that the spectral index values are robust, we require that the difference across the band is at least $3 \times$ the image rms noise level of $0.07 \mathrm{mJy} /$ beam. Therefore, as 7 per cent of the band is at least $0.21 \mathrm{mJy} /$ beam, then the total flux density must be at least $\sim 3 \mathrm{mJy} /$ beam. Hence, we removed all spectral index values for pixels with a flux density below this level ${ }^{3}$. This step allows us to have confidence that the spectral index values for the detected components are robust and correct. The $3 \mathrm{mJy} /$ beam cut off only leaves in-band spectral index values for components $\mathrm{C} 3, \mathrm{C} 4 \mathrm{E}$ and $\mathrm{C} 4 \mathrm{~W}$. We estimate uncertainties on the spectral index of each component by fitting a Gaussian to the pixel values in the spectral index image and report the full-width at half maximum value as the $1 \sigma$ error. The spectral indices of the components detected above the $3 \sigma$ level are shown in Table 1.

3 see the e-MERLIN webpage for a step-by-step discussion of how to perform this calculation: http : //www.e merlin.ac.uk/datared/CASA/Errors.html

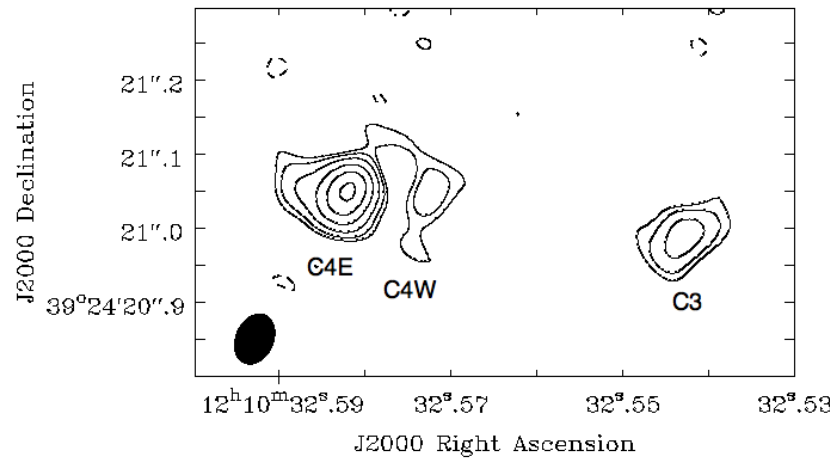

Figure 2. Archival MERLIN data from (Pedlar et al. 1993) of the central $0.50^{\prime \prime} \times 0.81^{\prime \prime}$ of NGC 4151 , corresponding to a linear scale of $45 \times 74 \mathrm{pc}^{2}$, re-imaged with a restoring beam of $0.05^{\prime \prime} \times 0.07^{\prime \prime}$ and a P.A. of $-24^{\circ}$ to match the e-MERLIN data, indicated by the filled ellipse in the corner of the image. The contour levels are set as the rms noise level, $0.5 \mathrm{mJy} /$ beam, $\times-3,3,5,9,16,25,36$, 49,64 .

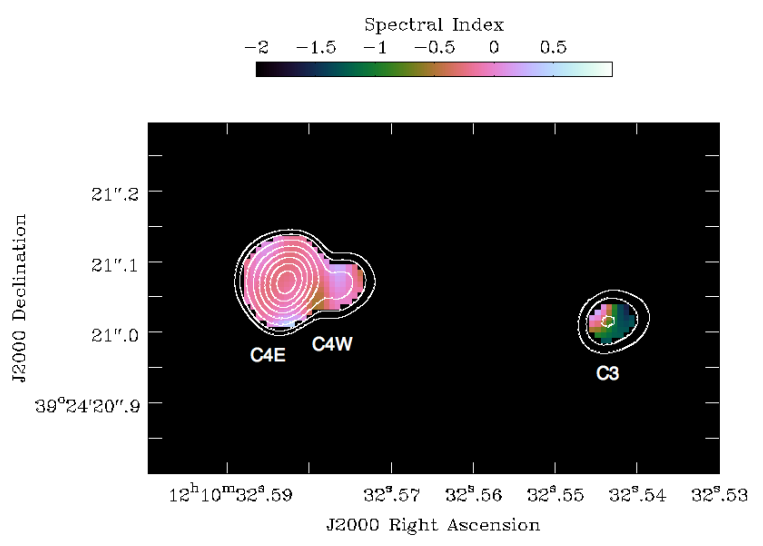

Figure 3. As in Fig. 2, but now presenting the new e-MERLIN $5 \mathrm{GHz}$ data of NGC 4151. The contour levels, beam size and P.A. are the same as in Fig. 2. The greyscale intensity image shows the spectral index image described in Section 2.4, with the intensity bar indicating the spectral index at the top.

\section{RESULTS}

We present the full resolution e-MERLIN image in Fig. 1. Listed above the components are the component names used in this paper, which are based off the names from previous studies at lower resolution (Carral et al. 1990; Williams et al. 2017). We add additional letters to distinguish Eastern and Western components. Below the components in Fig. 1, we show the component names used in VLBA studies at 
higher resolution (Ulvestad et al. 1998; Mundell et al. 2003). The increased sensitivity of the new e-MERLIN observations over the archival MERLIN data allows us to investigate additional compact components along the jet axis (see Section 4.2). We now break the radio jets into three parts, to describe their morphological properties, flux densities and spectra. The Western jet contains components $\mathrm{C} 1$ through $\mathrm{C} 2$, the nuclear region includes components $\mathrm{C} 3$ and $\mathrm{C} 4$, and the Eastern jet as components C5 to C6.

\subsection{The Western Jet}

Component A (also known as C1), is the first component of the Western jet, furthest away from the nucleus. It has a peak flux density of $0.66 \pm 0.08 \mathrm{mJy} /$ beam, and a similar integrated flux density, indicating that it is unresolved. Component $\mathrm{B}$ splits into two separate compact components in our $5 \mathrm{GHz}$ data, which we label $\mathrm{C} 2 \mathrm{~W}$ and $\mathrm{C} 2 \mathrm{E}$. A hint of this break can be seen in the $1.5 \mathrm{GHz}$ e-MERLIN images (Fig. 3 in Williams et al. 2017), but is strengthened by the appearance of two regions in the VLBA/VLA images at $21 \mathrm{~cm}$. The Western component $(\mathrm{C} 2 \mathrm{~W})$ is unresolved and is only detected at $\sim 4 \sigma$ at a peak flux density of $0.29 \pm 0.07 \mathrm{mJy} /$ beam in the e-MERLIN data. The Eastern component of $\mathrm{C} 2$ on the other hand is extended north-south, and has a peak flux density of $0.47 \pm 0.07 \mathrm{mJy} / \mathrm{beam}$. Due to the low flux density of all components in the Western jet, we were unable to ascertain a spectral index for these components.

\subsection{The Nuclear Region}

The $u v$-matched MERLIN and e-MERLIN images of the central core components $\mathrm{C} 3$ and $\mathrm{C} 4$ are presented in Fig. 2 and Fig. 3, respectively. The brightest features of both these components are detected in both the archival MERLIN and the new e-MERLIN $5 \mathrm{GHz}$ data. C3 (C) is slightly resolved in the east-west direction and it has a peak flux density of $4.56 \pm 0.26 \mathrm{mJy} /$ beam in the new e-MERLIN data, which represents a $2.7 \sigma$ decrease from the archival MERLIN data, when it was $6.32 \pm 0.61 \mathrm{mJy} /$ beam. The in-band e-MERLIN spectral index is $-1.19 \pm 0.76$, indicating it is likely spectrally steep, but the uncertainties are large.

Component $\mathrm{C} 4$ is separated into four distinct components in the $21 \mathrm{~cm}$ VLBA images: X, D (C4W) and E (C4E) and $\mathrm{F}$. We detect all bar component $\mathrm{F}$, which is likely connected with component $\mathrm{E}$ in the VLBA data (see Fig. 2 of Mundell et al. (2003)), but is faint, diffuse and possibly also resolved out in our e-MERLIN data. Moving west-to-east, component $\mathrm{X}$ is faint but detected at $\gtrsim 10 \sigma$, with a peak flux density of $0.92 \pm 0.08 \mathrm{mJy} /$ beam. It is slightly extended in the east-west direction. It should be noted that the full resolution images shown in Pedlar et al. 1993 hint at an extension that could plausibly be related to component X, but we do not detect this component in the re-reduced MERLIN images above a $3 \sigma$ threshold. This is likely due to the reduction in $u v$-range for our images. As such, it is not possible to investigate whether it has varied between epochs. Similarly, this source is below the cut-off level in the spectral index eMERLIN image, so we cannot draw any robust conclusions on the spectra in this component.

The AGN is thought to reside in Component $\mathrm{C} 4 \mathrm{~W}$
(D) (see Discussion), and it is detected in the MERLIN data $(3.82 \pm 0.54 \mathrm{mJy} /$ beam $)$ and the e-MERLIN data $(5.38 \pm 0.28 \mathrm{mJy} /$ beam $)$, indicating a tentative increase of $2.6 \sigma$ in flux density between epochs. This component also has a spectral index of $-0.02 \pm 0.34$, indicating it is spectrally flat. Component $\mathrm{C} 4 \mathrm{E}$ is the brightest of the three detected components at a peak flux density of $37.09 \pm 1.86$ mJy/beam. In the MERLIN data, this component was detected at a peak flux density of $19.35 \pm 1.10 \mathrm{mJy} /$ beam, which indicates that this component has nearly doubled in the 25 year period between the two epochs and represents a change of $8.2 \sigma$. The radio spectrum of this source $(-0.19 \pm 0.17)$ indicates that this component is possibly spectrally steeper than component $\mathrm{C} 4 \mathrm{~W}$.

\subsection{The Eastern Jet}

C5, the largest component from Mundell et al. (2003) is split into three components spanning $1^{\prime \prime}: \mathrm{G}, \mathrm{H}$ and $\mathrm{I}$ in the VLBA/VLA images. Component $\mathrm{G}$ is not detected in our eMERLIN images to a $3 \times$ rms-noise limit of $0.21 \mathrm{mJy} /$ beam, but it is one of the faintest and more diffuse components, which our data is less sensitive to. Component $\mathrm{H}$ separates into two components in our e-MERLIN images, which we delineate once more into east and west components. The Western component of $\mathrm{H}$ is detected at $0.49 \pm 0.08 \mathrm{mJy} /$ beam and the Eastern component has a peak flux density of $0.67 \pm 0.08 \mathrm{mJy} /$ beam. Finally, component I is detected also as a compact, unresolved point source with a peak flux density of $0.55 \pm 0.08 \mathrm{mJy} /$ beam. None of the components in the Eastern jet were detected in the re-analysed MERLIN data, and similar to the Western jet, and the flux densities in the e-MERLIN data are too low to reach a reliable conclusion for their spectra either.

\section{DISCUSSION}

Previous VLBI studies of NGC 4151 at GHz frequencies have suggested that the AGN resides unresolved inside either component C4E (Pedlar et al. 1993; Ulvestad et al. 1998) or C4W (Mundell et al. 1995; Ulvestad et al. 2005b). The first VLBI observations of NGC 4151 showed that component $\mathrm{E}$, unresolved inside $\mathrm{C} 4 \mathrm{E}$, was the brightest component at $1.5 \mathrm{GHz}$ and separates into several smaller components that are perpendicular to the overall jet axis (Ulvestad et al. 1998). Hence it was thought that the jet emerged from the AGN in component $\mathrm{E}$ and then bent at nearly right angles to form the rest of the observed jet. However, further VLBI studies at multiple frequencies showed that the radio spectral index of component E was steep and therefore unlikely to be the site of the AGN (Ulvestad et al. 2005b). In the same work, component $\mathrm{D}$, unresolved inside component $\mathrm{C} 4 \mathrm{~W}$, was shown to separate into three distinct components. The central component of D3, D3b, has a flat spectral index, a flux density of $3.0 \pm 0.4 \mathrm{mJy}$ and a brightness temperature, $\mathrm{T}_{\mathrm{b}}>10^{8} \mathrm{~K}$, indicative of an AGN. Furthermore, in HI studies of NGC 4151, Mundell et al. (1995) showed that the absorbing $\mathrm{HI}$ column towards component $\mathrm{E} / \mathrm{C} 4 \mathrm{E}$ was several orders of magnitude larger than that towards component $\mathrm{D} / \mathrm{C} 4 \mathrm{~W}$, indicating that component $\mathrm{E} / \mathrm{C} 4 \mathrm{E}$ lay behind the obscuring medium of the torus and therefore could not be the AGN. 


\begin{tabular}{|c|c|c|c|c|c|c|c|c|}
\hline Comp. & $\begin{array}{l}\text { e-MERLIN } \\
\text { Right } \\
\text { Ascension }\end{array}$ & $\begin{array}{l}\text { e-MERLIN } \\
\text { Declination }\end{array}$ & $\begin{array}{l}\text { e-MERLIN } \\
\text { Peak Flux } \\
\text { Density } \\
\text { mJy/beam }\end{array}$ & $\begin{array}{c}\text { e-MERLIN } \\
\text { Int Flux } \\
\text { mJy }\end{array}$ & $\begin{array}{l}\text { MERLIN } \\
\text { Peak Flux } \\
\text { Density } \\
\text { mJy/beam }\end{array}$ & $\begin{array}{c}\text { MERLIN } \\
\text { Int Flux } \\
\text { mJy }\end{array}$ & $\begin{array}{l}\text { Spectral } \\
\text { Index } \\
\alpha\end{array}$ & $\begin{array}{c}\text { Peak Flux } \\
\text { Density } \\
\text { Change } \\
(\sigma)\end{array}$ \\
\hline C1 (A) & 121032.441 & +392420.64 & $0.66 \pm 0.08$ & $0.70 \pm 0.15$ & - & - & - & - \\
\hline C2W (B) & 121032.491 & +392420.73 & $0.29 \pm 0.07$ & $0.34 \pm 0.14$ & - & - & - & - \\
\hline $\mathrm{C} 2 \mathrm{E}(\mathrm{B})$ & 121032.503 & +392420.90 & $0.47 \pm 0.07$ & $0.53 \pm 0.13$ & - & - & - & - \\
\hline C3 (C) & 121032.543 & +392421.02 & $4.56 \pm 0.26$ & $7.31 \pm 0.49$ & $6.32 \pm 0.61$ & $9.50 \pm 1.29$ & $-1.19 \pm 0.76$ & 2.7 \\
\hline $\mathrm{C} 4(\mathrm{X})$ & 121032.566 & +392421.05 & $0.92 \pm 0.08$ & $1.36 \pm 0.20$ & - & - & - & - \\
\hline $\mathrm{C} 4 \mathrm{~W}$ (D) & 121032.576 & +392421.07 & $5.38 \pm 0.28$ & $5.62 \pm 0.31$ & $3.82 \pm 0.54$ & $5.0 \pm 1.1$ & $-0.02 \pm 0.34$ & 2.6 \\
\hline $\mathrm{C} 4 \mathrm{E}(\mathrm{E})$ & 121023.583 & +392421.07 & $37.09 \pm 1.86$ & $43.70 \pm 2.19$ & $19.35 \pm 1.10$ & $26.4 \pm 1.7$ & $-0.19 \pm 0.17$ & 8.2 \\
\hline C5W (H) & 121032.653 & +392421.33 & $0.49 \pm 0.08$ & $1.10 \pm 0.25$ & - & - & & - \\
\hline $\mathrm{C} 5 \mathrm{E}(\mathrm{H})$ & 121032.662 & +392421.40 & $0.67 \pm 0.08$ & $0.86 \pm 0.16$ & - & - & & - \\
\hline C5 (I) & 121032.693 & +392421.39 & $0.55 \pm 0.08$ & $0.60 \pm 0.13$ & - & - & & - \\
\hline
\end{tabular}

Table 1. New e-MERLIN $5 \mathrm{GHz}$ positions, flux densities and spectral indices of all components identified in Fig. 3, as well as MERLIN flux densities for the detected components obtained from Fig. 2, all obtained from the uv-matched datasets. The uncertainties on the flux densities are found from the 2D Gaussian fitting procedure in CASA called IMFIT. We report the flux density uncertainties which include the flux density boot strapping uncertainty from the flux density calibrator 3C286, thought to be no more than 5 per cent in the MERLIN and e-MERLIN data, and the fitting uncertainty from IMFIT, added in quadrature. The flux density change is calculated using the equations in Zhou et al. (2006); Bruni et al. (2012). The positions listed in this table are typically accurate to a $\sim 10$ mas, or approximately 20 per cent of the synthesized beam.

They observed "banana-like" structures in components C4E and $\mathrm{C} 3$, indicative of interaction with dense clouds of gas in the emission-line region (ELR) close to the AGN. Thus the consensus now is that the AGN most likely resides in component $\mathrm{C} 4 \mathrm{~W}$ and that component $\mathrm{C} 4 \mathrm{E}$ is the site of the first jet component that is interacting with the ELR.

Our new e-MERLIN data show that the radio component C4W has not significantly varied over the course of 25 years $(2.6 \sigma$ change). Furthermore, it has a radio spectral index consistent with being an AGN, in agreement with the previous studies. The e-MERLIN data presented here are unable to challenge the interpretation of the AGN being in component $\mathrm{C} 4 \mathrm{~W}$.

\subsection{Explaining the variability in $\mathrm{C} 4 \mathrm{E}$}

Component $\mathrm{C} 4 \mathrm{E}$ has clearly doubled in flux density and is responsible for the majority of the flux density increase observed in the lower-resolution e-MERLIN studies. In addition $\mathrm{C} 4 \mathrm{E}$ has a slightly steeper radio spectral index, but is also consistent with being spectrally flat, given the uncertainties. We now consider what may be causing the change in flux density in $\mathrm{C} 4 \mathrm{E}$, focussing on processes not related to accretion. These include shock radio emission from interaction of the jet with dense clouds in the extended emission line region (ELR, e.g. Middelberg et al. 2007), or continued acceleration of particles along the jet (e.g. Blandford et al. 2019). If the flux density increase in $\mathrm{C} 4 \mathrm{E}$ is interpreted as due to shocks from jet-ELR interaction, multi-frequency VLBI observations should reveal a significantly brighter component, an extended morphology to that observed in Ulvestad et al. (2005a), and possibly a flat spectrum 'hot spot' of emission with a steeper surrounding spectral index. If the observed flux density change is due to further particle acceleration in the jet, we would expect a flat spectrum source. We note that our e-MERLIN data is not of high enough resolution to distinguish between these two scenarios. Thus, VLBI observations are the only way to break the degeneracy of the exact cause of the change in flux density in this component, assuming it is not directly related to the AGN. For this purpose, we have obtained proprietary VLBI and EVN data (PI: Panessa, project EP113) which we will publish in a subsequent paper (Panessa et al. in prep.).

\subsection{Testing the adiabatic expansion hypothesis in component C3}

Component $\mathrm{C} 3(\mathrm{C})$ is the only component in the data to have decreased in flux density between the two epochs of data, from $6.32 \pm 0.61$ to $4.56 \pm 0.26 \mathrm{mJy} /$ beam, though we note that this only represents a change of $2.7 \sigma$. Williams et al. (2017) attribute the decrease in flux density to adiabatic expansion of component $\mathrm{C} 3$, as the timescales of simple radiative losses from synchrotron emission were too long to explain the flux density decrease. On the assumption that the decrease in flux density is real, we perform the same analysis here, first working out the equipartition magnetic field, and subsequently the synchrotron cooling timescale and compare that with the predicted flux density decrease from adiabatic expansion. A description of this method is found in Section 3.4 and 3.5 of Williams et al. (2017). We note that while the two epochs have slightly different central frequencies, this difference would only lead to a 1 per cent change in flux density.

Using the component size for C3 (58.1mas $\times 20.6 \mathrm{mas})$ from the present $5 \mathrm{GHz}$ e-MERLIN observations, and following the method outlined in Williams et al. (2017), we derive a magnetic field strength of $0.9 \mathrm{mG}$ and a synchrotron cooling timescale $\left(\tau \propto \mathrm{B}^{-1.5} v^{-0.5}\right)$ of $\sim 2.17 \times 10^{4}$ years. We note, however, that a tighter limit of 700 years is still provided by the VLBI observations (Ulvestad et al. 1998; Mundell et al. 2003; Ulvestad et al. 2005a) which were discussed in Williams et al. (2017). We therefore reaffirm that synchrotron cooling cannot explain the flux density decrease in component C3.

We now turn to understanding the flux density de- 
crease from adiabatic expansion of component C3. Following Scheuer \& Williams (1968), the change in flux density is related to a simple linear expansion factor, $\mathrm{F}$, and the spectral index, $\alpha$, as $\mathrm{F}^{4 \alpha-2}$. Therefore, to explain the change in flux density of $\sim 26$ per cent between the two epochs, we require a linear expansion factor of $\mathrm{F} \sim 1.04-1.05$. which is a similar value to that calculated by Williams et al. (2017), and yields an expansion velocity of $\sim 1700 \mathrm{~km} \mathrm{~s}^{-1}$, qualitatively the same as that calculated by Williams et al. (2017).

\subsection{NGC 4151 and jets in other low luminosity AGN}

Radio jets are seen in other low luminosity AGN, e.g. NGC 1052 (Baczko et al. 2016, 2019; Nakahara et al. 2020). However there are significant differences between both the jet properties and the host galaxy properties between NGC 4151 and these other AGN. For example, in NGC 1052 jet speeds of $\sim 0.34 \mathrm{c}$ (western jet) and $\sim 0.53 \mathrm{c}$ (eastern jet) are found whereas in NGC 4151 no detectable movement is found (Ulvestad et al. 2005a). Also in NGC 1052, the overall morphologies change over a four year period and flux density variations in jet components are seen on that timescale (Baczko et al. 2016, 2019). Moreover the radio luminosity of NGC 1052 is $100 \times$ that of NGC 4151 and is unequivocally a radio-loud object (Kadler et al. 2004). However although NGC 4151 and NGC 1052 have similar black hole masses $\left(\log \left(\mathrm{M}_{\mathrm{BH}}\right)=7.7\right.$ and 8.2 , respectively $)$, they reside in very different host galaxies: NGC 4151 is a Seyfert 1.5 spiral galaxy whereas NGC 1052 is classified as an elliptical LINER galaxy. Thus the majority of the nuclear emission in NGC 4151 is probably powered by an efficient accretion flow whereas that in NGC 1052 is likely powered by an inefficient ADAF flow which more naturally launches jets (e.g. see Narayan \& Yi 1994; Yuan \& Narayan 2014, and references therein). For a Seyfert galaxy, NGC 4151 is particularly radio luminous (Nagar et al. 2005). Although usually classed as 'radio-quiet', it is occasionally classed as radio loud (Zdziarski et al. 2000; Kadler et al. 2004). It also has a relatively low accretion rate $(\sim 2$ per cent Eddington (McHardy et al. 2018) for a Seyfert galaxy and so may be a transition object between radio loud LINERs and radio quiet Seyferts (Mahmoud \& Done 2020).

\subsection{Resolving new components along the jet}

The higher sensitivity of our new $5 \mathrm{GHz}$ e-MERLIN images have allowed us to clearly resolve compact radio components along the jet, such as C1, C2, C5 and C6, seen previously in the lower-resolution studies. The images presented in Pedlar et al. 1993 are more sensitive to the diffuse emission at $5 \mathrm{GHz}$, but, due to the higher frequency and lack of $u v$ spacings below $400 \mathrm{k} \lambda$ in our new e-MERLIN images, the diffuse emission is resolved out. It is likely that the jet is continuous, as seen in the $21 \mathrm{~cm}$ VLBA and phased VLA images of Mundell et al. 2003, which are sensitive to a similar angular scale (40mas synthesized beam) as our data presented here along the jet spine in Fig. 1. Hence, our images show compact hot spots along the jet spine, which are embedded in much larger diffuse components which are resolved out in our images.
As the $21 \mathrm{~cm}$ Mundell et al. (2003) data are of a similar resolution to our $5 \mathrm{GHz}$ e-MERLIN data, we are able to reach general conclusions about the individual components along the jet axis, by considering the radio spectra between the two datasets. All of the components in Fig. 1 appear to align closely with components in the Mundell et al. (2003) image and have flux densities in the range $\sim 1-2 \mathrm{mJy} /$ beam. Given that the peak flux densities in all of these components are $1 \mathrm{mJy}^{-1}$ beam $^{-1}$ in the $5 \mathrm{GHz}$ e-MERLIN data, this implies a steeper spectral index $(\alpha<0)$. For example, for the brightest and faintest e-MERLIN components in either the Western or Eastern jets, C5E and C2W respectively, the spectral index ranges from $\sim-0.3$ to $\sim-1.0$. These values are only representative, but are qualitatively consistent with those found by Williams et al. (2017) for the same components. The components along the jet spine are likely impact regions where the jet interacts with the extended emission line region and their steeper spectral indices are likely indicative of synchrotron ageing due to radiative losses (Condon \& Ransom 2016).

\section{CONCLUSIONS}

We present new $5 \mathrm{GHz}$ e-MERLIN observations of the radio-quiet Seyfert galaxy NGC 4151, achieving $70 \mu \mathrm{Jy}$ rmssensitivity and $\sim 0.05^{\prime \prime}$ resolution along the jet axis. We find that the Eastern component, $\mathrm{C} 4 \mathrm{E}$, is responsible for the majority of the increase in flux density in the nucleus of NGC 4151 reported in Williams et al. (2017). Component $\mathrm{C} 4 \mathrm{~W}$, generally favoured as the most likely location of the AGN, has not varied significantly between epochs (2.6 $\sigma$ increase). We compare the flux density to archival MERLIN data from 1991 (Pedlar et al. 1993) and find that when comparing like-for-like $u v$-coverage, component $\mathrm{C} 4 \mathrm{E}$ has increased by a factor of $\sim 2$, representing a $8.2 \sigma$ increase. Furthermore, we calculate in-band spectral indices for all of the detected e-MERLIN components at $5 \mathrm{GHz}$, which show that component $\mathrm{C} 4 \mathrm{~W}$ is consistent with being spectrally flat ( $\alpha=-$ $0.02 \pm 0.34)$, whereas component $\mathrm{C} 4 \mathrm{E}$ is slightly steeper $(\alpha=-$ $0.19 \pm 0.17)$ and C3 is spectrally steeper still $(\alpha=-1.19 \pm 0.76)$. Owing to the improved sensitivity of e-MERLIN, component $\mathrm{X}$ is detected in the nuclear region for the first time at $5 \mathrm{GHz}$, but due to the low flux density, we are unable to produce a reliable spectral index image or variability constraints.

We interpret the increase in flux density of component $\mathrm{C} 4 \mathrm{E}$ as most likely due to a region of particle acceleration or continued jet-ELR interaction causing shock excited radio emission. This scenario will be tested using further highresolution radio interferometers such as the VLBA and EVN. We emphasise that multiple frequency observations are best for future radio studies, as the radio spectral index, $\alpha$, will be important in discerning the cause of the increase in radio emission. We also test the adiabatic expansion hypothesis suggested by Williams et al. (2017), and find it consistent with our new data, though we note that the flux decrease of this component is only significant to $2.7 \sigma$. Furthermore, to probe the compact regions further along the jet spine than previously performed at this frequency with the MERLIN interferometer. By comparing to the VLBA/VLA data of Mundell et al. (2003), with a similar angular resolution as e-MERLIN at $5 \mathrm{GHz}$, we are able to give rough estimates 
on the spectral index for these components, which indicate that these are likely hotspots of interaction regions along the jet spine. These hotspots are likely embedded within a more diffuse structure as the jet of NGC 4151 is continuous as shown by Mundell et al. (2003) and other studies with higher sensitivity from longer observations. The inclusion of the Lovell and possibly the VLA would be needed to explore the diffuse emission futher with e-MERLIN.

This work emphasises the need for high-resolution studies to fully understand the nature of variability in LLAGN. Previous radio variability studies of LLAGN have had mixed results (Wrobel 2000; Nagar et al. 2002; Anderson \& Ulvestad 2005; Mundell et al. 2009; Jones et al. 2011; Bell et al. 2011; Baldi et al. 2015), providing evidence for and against nuclear variability. For the most part, these studies have assumed that the variability is due only to the AGN. However, our observations of NGC 4151 show the need for high-resolution radio interferometry in nearby LLAGN as it is plausible that previous studies of changes in flux density are are potentially unrelated to AGN activity, and can be attributed to regions in the jet. Such variability caused by indirect AGN activity can have important implications for waveband scaling relationships for AGN and therefore further investigation is needed to fully understand the origin of variability in radio-quiet AGN.

\section{ACKNOWLEDGEMENTS}

We thank the anonymous reviewer for their comments and revisions, which greatly improved the quality of this manuscript. We acknowledge funding from the Mayflower Scholarship from the University of Southampton afforded to DW to complete this work. This work was supported by the Oxford Centre for Astrophysical Surveys, which is funded through generous support from the Hintze Family Charitable Foundation. The research leading to these results has received funding from the European UnionâĂŹs Horizon 2020 Programme under the AHEAD project (grant agreement n. 654215). This publication has also received funding from the European Union's Horizon 2020 research and innovation programme under grant agreement No 730562 [RadioNet]. IMcH thanks the Royal Society for the award of a Royal Society Leverhulme Trust Senior Research Fellowship. RDB and IMcH also acknowledge the support of STFC under grant [ST/M001326/1]. FP acknowledges support from grant PRIN-INAF SKA-CTA 2016. GB acknowledges financial support under the INTEGRAL ASI-INAF agreement 2013-025-R1. JHK acknowledges financial support from the European Union's Horizon 2020 research and innovation programme under Marie Skłodowska-Curie grant agreement No 721463 to the SUNDIAL ITN network, and from the Spanish Ministry of Science, Innovation and Universities (MCIU) and the European Regional Development Fund (FEDER) under the grant with reference AYA2016$76219-\mathrm{P}$, from IAC project $\mathrm{P} / 300724$, financed by the Ministry of Science, Innovation and Universities, through the State Budget and by the Canary Islands Department of Economy, Knowledge and Employment, through the Regional Budget of the Autonomous Community, and from the Fundación BBVA under its 2017 programme of assistance to scientific research groups, for the project "Using machine-learning techniques to drag galaxies from the noise in deep imaging". DMF wishes to acknowledge funding from an STFC Q10 consolidated grant [ST/M001334/1]. EB and JW acknowledge support from the UK's Science and Technology Facilities Council [grant number ST/M503514/1] and [grant number ST/M001008/1], respectively. JM acknowledges financial support from the State Agency for Research of the Spanish MCIU through the "Center of Excellence Severo Ochoa" award to the Instituto de Astrofísica de Andalucía (SEV-2017-0709) and from the grant RTI2018096228-B-C31 (MICIU/FEDER, EU). CGM acknowledges financial support from STFC. We also acknowledge Jodrell Bank Centre for Astrophysics, which is funded by the STFC. eMERLIN and formerly, MERLIN, is a National Facility operated by the University of Manchester at Jodrell Bank Observatory on behalf of STFC. MP acknowledge the support from the Royal Society Newton International Fellowship.

\section{REFERENCES}

Anderson J. M., Ulvestad J. S., 2005, ApJ, 627, 674

Baczko A.-K., et al., 2016, A\&A, 593, A47

Baczko A.-K., Schulz R., Kadler M., Ros E., Perucho M., Fromm C. M., Wilms J., 2019, A\&A, 623, A27

Baldi R. D., Behar E., Laor A., Horesh A., 2015, MNRAS, 454, 4277

Baldi R. D., et al., 2018, MNRAS, 476, 3478

Begelman M. C., Blandford R. D., Rees M. J., 1984, Reviews of Modern Physics, 56, 255

Bell M. E., et al., 2011, MNRAS, 411, 402

Blandford R., Meier D., Readhead A., 2019, ARA\&A, 57, 467

Bruni G., et al., 2012, A\&A, 542, A13

Carral P., Turner J. L., Ho P. T. P., 1990, ApJ, 362, 434

Condon J. J., Ransom S. M., 2016, Essential Radio Astronomy

Fabian A. C., 2012, ARA\&A, 50, 455

Falcke H., Markoff S., 2000, A\&A, 362, 113

Fanaroff B. L., Riley J. M., 1974, MNRAS, 167, 31P

Gallimore J. F., Baum S. A., O'Dea C. P., 2004, ApJ, 613, 794

Ghisellini G., Haardt F., Matt G., 2004, A\&A, 413, 535

Giroletti M., Panessa F., Longinotti A. L., Krongold Y., Guainazzi M., Costantini E., Santos-Lleo M., 2017, A\&A, 600, A 87

Hönig S. F., Watson D., Kishimoto M., Hjorth J., 2014, Nature, 515,528

Hufnagel B. R., Bregman J. N., 1992, ApJ, 386, 473

Jones S., McHardy I., Moss D., Seymour N., Breedt E., Uttley P., Körding E., Tudose V., 2011, MNRAS, 412, 2641

Kadler M., Ros E., Kerp J., Kovalev Y., Zensus J. A., 2004, in European VLBI Network on New Developments in VLBI Science and Technology. pp 23-26 (arXiv:astro-ph/0409300)

Kellermann K. I., Sramek R., Schmidt M., Shaffer D. B., Green R., 1989, AJ, 98, 1195

Laor A., Baldi R. D., Behar E., 2019, MNRAS, 482, 5513

Mahmoud R. D., Done C., 2020, MNRAS, 491, 5126

McHardy I. M., et al., 2018, MNRAS, 480, 2881

Middelberg E., Agudo I., Roy A. L., Krichbaum T. P., 2007, MNRAS, 377, 731

Morganti R., Fogasy J., Paragi Z., Oosterloo T., Orienti M., 2013, Science, 341, 1082

Mundell C. G., Pedlar A., Baum S. A., O'Dea C. P., Gallimore J. F., Brinks E., 1995, MNRAS, 272, 355

Mundell C. G., Wrobel J. M., Pedlar A., Gallimore J. F., 2003, ApJ, 583, 192

Mundell C. G., Ferruit P., Nagar N., Wilson A. S., 2009, ApJ, 703,802 
Nagar N. M., Falcke H., Wilson A. S., Ho L. C., 2000, ApJ, 542, 186

Nagar N. M., Falcke H., Wilson A. S., Ulvestad J. S., 2002, A\&A, 392,53

Nagar N. M., Falcke H., Wilson A. S., 2005, A\&A, 435, 521

Nakahara S., Doi A., Murata Y., Nakamura M., Hada K., Asada K., Sawada-Satoh S., Kameno S., 2020, AJ, 159, 14

Narayan R., Yi I., 1994, ApJ, 428, L13

Offringa A. R., van de Gronde J. J., Roerdink J. B. T. M., 2012, A\&A, 539

Panessa F., Baldi R. D., Laor A., Padovani P., Behar E., McHardy I., 2019, Nature Astronomy, 3, 387

Pedlar A., Kukula M. J., Longley D. P. T., Muxlow T. W. B., Axon D. J., Baum S., O'Dea C., Unger S. W., 1993, MNRAS, 263,471

Rampadarath H., et al., 2018, MNRAS, 476, 2876

Scheuer P. A. G., Williams P. J. S., 1968, ARA\&A, 6, 321

Shakura N. I., Sunyaev R. A., 1973, A\&A, 24, 337

Ulvestad J. S., Ho L. C., 2001, ApJ, 562, L133

Ulvestad J. S., Roy A. L., Colbert E. J. M., Wilson A. S., 1998, ApJ, 496, 196

Ulvestad J. S., Wong D. S., Taylor G. B., Gallimore J. F., Mundell C. G., 2005a, AJ, 130, 936

Ulvestad J. S., Wong D. S., Taylor G. B., Gallimore J. F., Mundell C. G., 2005b, AJ, 130, 936

Urry C. M., Padovani P., 1995, PASP, 107, 803

Westcott J., et al., 2017, MNRAS, 467, 2113

Williams D. R. A., et al., 2017, MNRAS, 472, 3842

Williams D. R. A., et al., 2019, MNRAS, 486, 4962

Wrobel J. M., 2000, ApJ, 531, 716

Yuan F., Narayan R., 2014, ARA\&A, 52, 529

Zdziarski A. A., Poutanen J., Johnson W. N., 2000, ApJ, 542, 703

Zhou H., Wang T., Wang H., Wang J., Yuan W., Lu Y., 2006, ApJ, 639, 716

This paper has been typeset from a $\mathrm{T}_{\mathrm{E}} \mathrm{X} / \mathrm{LAT}_{\mathrm{E}} \mathrm{X}$ file prepared by the author. 